\title{
Patency and flow velocity profiles in the internal carotid artery assessed by digital subtraction angiography and Doppler studies three months following endarterectomy
}

\author{
D RUSSELL, S J BAKKE, J WIBERG, P NAKSTAD, R NYBERG-HANSEN \\ From the Departments of Neurology, Neurosurgery, and Radiology, Section of Neuroradiology, Rikshospitalet, \\ The National Hospital, The University of Oslo, Norway
}

SUMMARY Pulsed Doppler spectral analysis and intravenous digital subtraction angiography have been carried out in 55 patients, three months after a total of 60 carotid endarterectomies. Five patients were operated on both sides and there were no re-operations. There were no re-stenoses and only one of the 60 internal carotid arteries was occluded. Spectral spreading of the Doppler signal at the site of the endarterectomy was found in $52 \%$ of the internal carotid arteries, whereas wall irregularities were demonstrated in $25 \%$ by intravenous digital subtraction angiography. A stenosis or occlusion was found in $11(18 \%)$ of the 60 ipsilateral external carotid arteries.

Patency of the internal carotid artery (ICA) is one of the principal objectives of carotid endarterectomy. Until recently the occurrence of postoperative stenosis or occlusion following endarterectomy has been suggested by the appearance of a bruit or a neurological event. Although conventional arteriography can define the changes occurring postoperatively, the potential risks of this examination make its use in the follow-up of asymptomatic individuals unacceptable.

The purpose of this paper is to report our experience with pulsed Doppler spectral analysis and intravenous digital subtraction angiography (IVDSA) used to determine the status of the carotid artery three months following endarterectomy.

\section{Materials and methods}

Fifty-five consecutive patients were examined three months following a total of 60 carotid endarterectomies. Five patients were operated on both sides and there were no reoperations. The operations were performed because of carotid artery stenosis which had caused transient ischaemic attacks or previous completed strokes. The stenoses were assessed by both conventional arteriography and Doppler

Address for reprint requests: David Russell, M.D. Department of Neurology, Rikshospitalet, The National Hospital, The University of Oslo, 0027 Oslo 1, Norway.

Received 15 November 1984 and in revised form 21 May 1985. Accepted 28 May 1985 examination pre-operatively. ${ }^{1}$ Fifty-nine of the 60 stenoses reduced the diameter of the carotid artery by $50 \%$ or more. In the remaining stenosis severe ulceration was present.

Carotid bifurcation endarterectomy was performed using standard vascular surgical technique, particular attention being paid to the removal of the entire plaque and the avoidance of intimal flaps. The arteriotomies were closed using 5-0 polypropylene sutures. All patients were anticoagulated intraoperatively with 5000 or 7500 IU of heparin. A temporary inlying shunt for cerebral protection was used routinely during the endarterectomy. ICA blood flow was measured using an electromagnetic flowmeter before and immediately after the endarterectomy ${ }^{2}$.

A clinical neurological examination was carried out on all patients three months after the operation, prior to the Doppler and IVDSA examinations.

All 55 patients were examined by pulsed Doppler spectral analysis. IVDSA was considered to be contraindicated in three patients owing to known contrast allergy and in one patient to congestive heart failure. Informed consent was given by all patients prior to these examinations. The Doppler apparatus and spectral analyser (Vingmed Inc, Oslo) have been described in detail previously. ${ }^{1}$ The Doppler system was used with a $5 \mathrm{MHz}$ frequency in pulsed mode, with a sample volume which can be approximated to a cylinder with a diameter and length of $4 \mathrm{~mm}$. A spectral analysis of the Doppler signal based on the Chirp-Z-transform was displayed in real time on a monitor and could be written out directly on a fiberoptic recorder, the Doppler spectrum being presented as a grey-scale printout.

In assessing the Doppler findings particular attention was paid to the Doppler spectrum in the acceleration phase of 
Table 1 Doppler and IVDSA findings in the ICA three months after endarterectomy.

Internal carotid artery

\begin{tabular}{|c|c|c|c|c|}
\hline Doppler findings & $\begin{array}{l}\text { Normal } \\
28\end{array}$ & $\begin{array}{l}\text { Spectral spreading } \\
31 \\
\text { Wall irregularities }\end{array}$ & $\begin{array}{l}\text { Occlusion } \\
1\end{array}$ & $\begin{array}{l}\text { Total } \\
60\end{array}$ \\
\hline IVDSA findings* & 41 & 14 & 1 & 56 \\
\hline
\end{tabular}

*Not carried out in 4 patients because of contra-indications.

systole. ${ }^{1}$ A lesion was diagnosed when the Doppler spectrum in a localised area of the vessel changed such that frequencies could clearly be seen in both the upper and lower parts of the spectrum in the acceleration phase of systole. Relative changes in the Doppler spectrum in the deceleration phase of systole, or in diastole were not assessed as being due to a lesion of the vessel. When maximum velocity at the site of the spectral spreading was not increased by more than $10 \%$, compared to the maximum velocity downstream in the internal carotid artery (ICA), at the angle of the jaw, a diagnosis of stenosis less than $10 \%$ (that is wall irregularities) was made. When the maximum velocity at the site of the spectral spreading $\left(V_{1}\right)$ was increased by more than $10 \%$, compared to the maximum velocity downstream in the ICA at the angle of the jaw $\left(\mathrm{V}_{2}\right)$, the percentage stenosis was calculated as follows: Stenosis (Doppler) $=\left(1-\mathrm{V}_{2} / \mathrm{V}_{1}\right) \times 100 \%$. An increase in maximum velocity without spectral spreading in the acceleration phase of systole was not assessed as being due to a lesion of the vessel.

The IVDSA examinations were carried out using an Angiotron (Siemens, Erlangen). This apparatus carries out a digital subtraction in a real-time continuous mode (50 frames/s), with a matrix of $512 \times 512$, and a 12 bits memory depth. The input field of the image intensifier was six inches $(15 \mathrm{~cm})$. Postprocessing was routinely performed so that optimal images could be assessed. Using the Seldinger technique, a 6 French, straight, polyethylene catheter with sideholes, was introduced into an antecubital vein, and positioned under fluoroscopy in the superior vena cava just above the right atrium. Forty millilitres of Omnipaque, $350 \mathrm{mg}$ iodine per $\mathrm{ml}$ (Nyegaard \& Co., Oslo) were injected at a rate of $15 \mathrm{ml} / \mathrm{s}$. Routine views were made of the carotid bifurcations at an angle of $65-70^{\circ}$ in right anterior oblique and left anterior oblique projections.

\section{Results}

Three patients developed a transient hemiparesis contralateral to the endarterectomy in the immediate postoperative period. All of these three patients had

Table 2 Doppler and IVDSA findings in the ipsilateral ECA three months after a total of 60 carotid endarterectomies.

External carotid artery

\begin{tabular}{lclc}
\hline & Doppler & IVDSA* \\
Stenosis & Occlusion & Stenosis & Occlusion \\
7 & 4 & 6 & 4 \\
\hline
\end{tabular}

*Not carried out in four patients because of contra-indications. generalised atherosclerosis of the precerebral vessels and in two the symptoms developed during a period with labile blood pressure. Doppler examination on the following day showed occlusion of the relevant ICA in one of these patients. Clinical neurological examination three months later showed only slight central motor signs in all three patients. A fourth patient experienced two transient ischaemic attacks in the relevant cerebral hemisphere, two months following the operation. A clinical neurological examination in this patient one month later showed similar findings to those found prior to the operation. The remaining patients had no relevant symptoms in the first three postoperative months, and no new neurological signs were detected on clinical neurological iv examination.

A cervical bruit was heard at the site of the endarterectomy in $11(18 \%)$ patients, all of whom had 을 also had a bruit preoperatively. None of thes patients had a stenosis of the relevant ICA, but fow TI $(36 \%)$ had a stenosis of the ipsilateral external carotid 음 artery (ECA).

The Doppler and IVDSA findings in the ICA thre months following endarterectomy are shown in tab $\overrightarrow{0}$ 1. Thirty-one $(52 \%)$ of the 60 Doppler examinations showed spreading of the Doppler spectrum at the site of the endarterectomy, with frequencies in both the upper and lower part of the spectrum in the acceleration phase of systole. The maximum velocity, however, was not increased by more than $10 \%$, when compared to the maximum velocity, downstream in the ICA, at the angle of the jaw. Occlusion of the one $\bar{c}$ ICA was also demonstrated by Doppler examination.

Abnormal Doppler findings, which were not present prior to endarterectomy, were also found in $11(18 \%)$ of the 60 ECAs, ipsilateral to the endarterectomy. Seven had a stenosis at their origin and four vessels were occluded (table 2).

Eleven patients had also been examined by Doppler examination in the first week following $\delta$ endarterectomy. Nine $(82 \%)$ of these eleven Doppler $₹$ examinations showed spectral spreading at the site of $\mathrm{O}$ the endarterectomy. When Doppler examination was repeated three months later (fig.1), however, spectral o spreading was no longer present in five of these ICAs.

Fourteen $(25 \%)$ of the 56 IVDSA examinations $O$ 


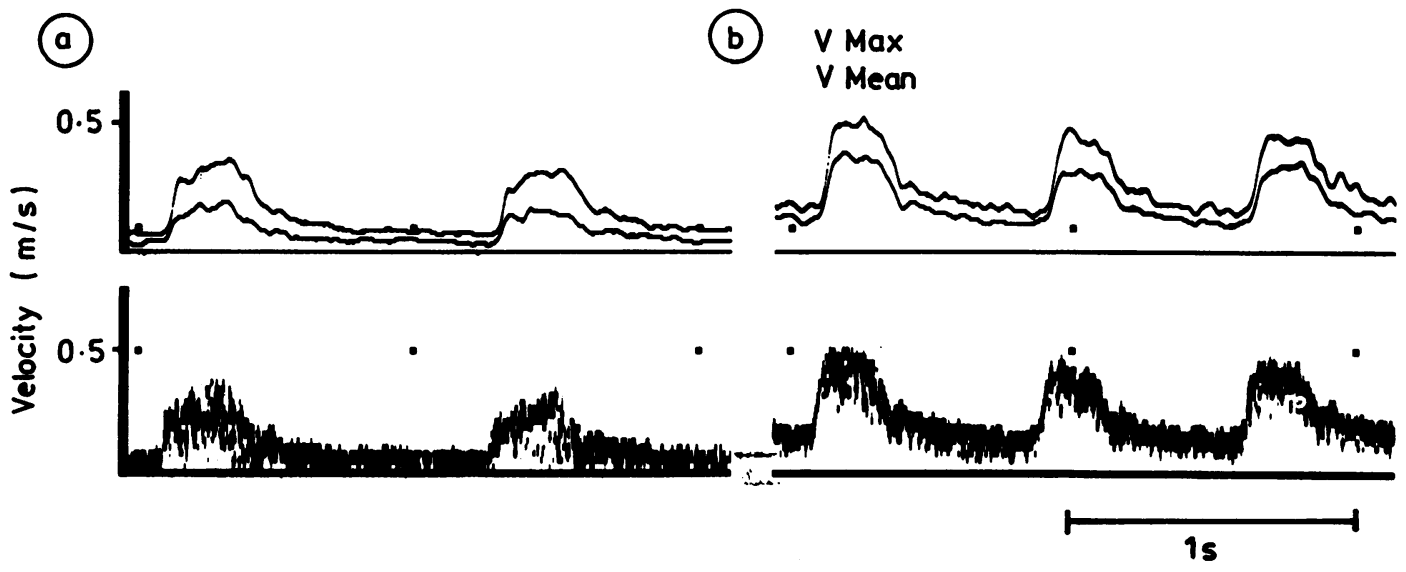

Fig 1 Spectral spreading of the Doppler signal in an ICA at the site of an endarterectomy, I week postoperatively (a). This spectral spreading is no longer present three months later $(b)$.

showed wall irregularities at the site of the endarterectomy, that is, a diameter reduction of less than $10 \%$ (fig 2 ) and one ICA was occluded. Abnormal findings not present before operation were also found in $10(18 \%)$ of the 56 ECAs ipsilateral to the endarterectomy by IVDSA. A stenosis of the origin of the ECA was found in six vessels and four vessels were occluded (table 2).

Of eight patients also examined by IVDSA in the first week following endarterectomy, three (38\%) showed wall irregularities at the site of the endarterectomy. Two of these three vessels showed normal findings when re-examined by IVDSA three months later (fig 3).

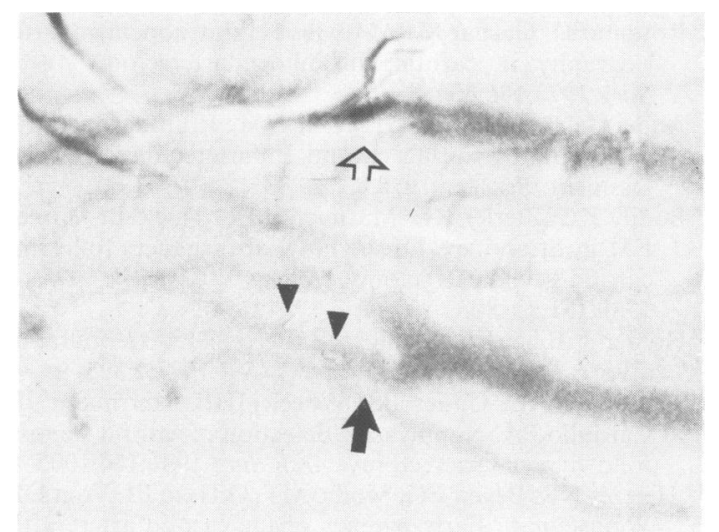

Fig 2 IVDSA showing wall irregularities after endarterectomy of the right ICA (closed arrow), artifacts due to swallowing at the left bifurcation (open arrow) and clips (small arrowheads).

\section{Discussion}

Although the incidence of stroke following carotid endarterectomy is low at most surgical centres, the immediate complication rate does not necessarily reflect the technical results of this operation. Patients may develop a stroke in the early postoperative period due to microembolism, with normal findings on subsequent arteriography, while others develop an occlusion at the ICA which is asymptomatic due to a compensatory increase in collateral circulation. ${ }^{34}$

In this study, there were no re-stenoses and only one of the 60 ICAs became occluded in the first three

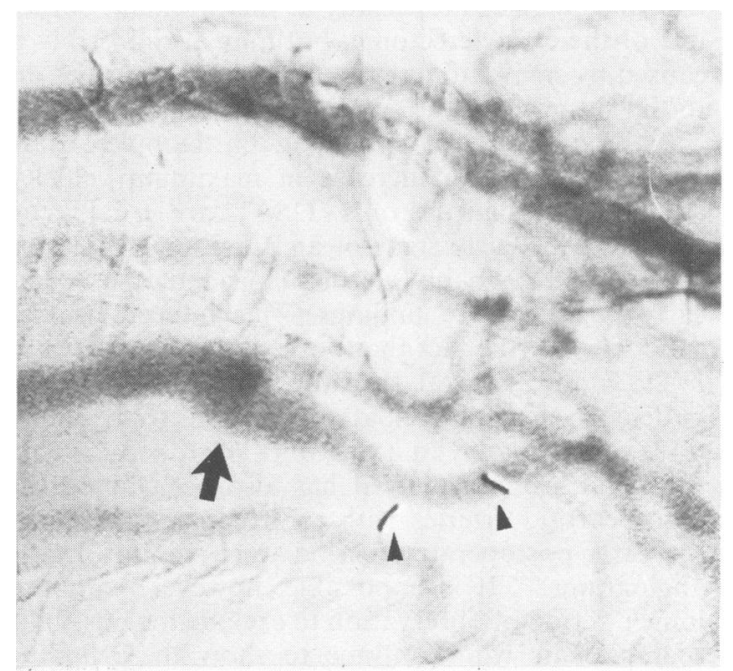

Fig 3 IVDSA showing smooth walls in the left ICA after endarterectomy (arrow) and clips (small arrowheads). 
months following endarterectomy. This occlusion was found in a patient who developed a transient hemiplegia a few hours after endarterectomy. Although three other patients developed a transient hemiplegia, and one patient two transient ischaemic attacks, none of them had restenosis or occlusion of the relevant ICA.

Previous reports regarding the early incidence of re-stenosis or occlusion of the ICA after endarterectomy demonstrated by conventional arteriography, IVDSA or Doppler examination, have shown considerable variation..$^{3-12}$ It is therefore emphasised that attention to surgical detail is the key to successful carotid reconstruction. ${ }^{11}$ We also stress the importance of complete removal of the atheromatous lesion with extension of the arteriotomy into the normal carotid lumen, and the avoidance of intimal flaps.

We have previously found that the pulsed Doppler spectral analysis system used in this study is accurate in the detection of lesions of the carotid artery, especially those which reduce the diameter by more than $25 \% .^{1}$ In this study the Doppler examinations showed spectral spreading without an increase in maximum velocity, at the site of the endarterectomy in approximately $50 \%$ of the ICAs. On IVDSA examination, however, half of these vessels showed normal findings. This suggests that the velocity profile in the endarterectomised segment of these vessels had not returned to normal, even though no morphological changes were detected by IVDSA.

Wall irregularities were seen in the ICA at the site of the endarterectomy in $25 \%$ of the IVDSA examinations. These findings are probably in most cases due to small steps especially at the proximal borderline of the endarterectomy, but may also possibly be caused by small intima remnants or irregularities due to the sutures.

Although spectral spreading on Doppler examination without an increase in maximum velocity ${ }^{1}$ and wall irregularities on IVDSA ${ }^{13}$ are usually considered to be early signs of an atheromatous lesion, they should probably also be considered as normal early postoperative findings. This interpretation is supported by the fact that both wall irregularities on IVDSA and spectral spreading were seen in some patients in the first week after endarterectomy but not three months later. In a review of postoperative conventional arteriography it has also been noted that many carotid arteries with an irregular wall during the early postoperative period were smooth in later angiograms. ${ }^{49}$ It is impossible, however, without a longer period of observation to exclude the possibility that patients who continue to show these findings may have a greater risk of recurrent stenosis.

Stenosis or occlusion of the ipsilateral ECA was found in approximately $20 \%$ of the Doppler and IVDSA examination. This is due to technical difficulties in the management of the distal end of the endarterectomy in the ECA so that intima flaps are $z$ more liable to cause stenosis or occlusion of this ves- $\stackrel{\mathbb{D}}{\subset}$ sel. Although a cervical bruit was heard on the side of the endarterectomy in $11(18 \%)$ of the cases, this clinical finding was not indicative of a restenosis of the ICA. Four of these patients had a stenosis of the rele-CD vant ECA, and in the remainder no explanation for ș the cervical bruit was found.

In conclusion, this study has shown that Doppler examination and IVDSA are well suited for assessment of the results of carotid endarterectomy, providing both haemodynamic and morphological informa-o tion. It is therefore now possible to assess the early as $\underline{\bar{D}}$ well as the long-term results of this operation without $\frac{\bar{m}}{\frac{\bar{g}}{}}$ subjecting the patient to the potential complications $\stackrel{\mathbb{\Omega}}{\varrho}$ of conventional arteriography.

\section{References}

${ }^{1}$ Russell D, Lindegaard K-F, Nakstad P, Nyberg-Hansen R, Øygarden KG. Detection of carotid occlusive disease by pulsed Doppler spectral analysis. J Neurol Neurosurg Psychiatry 1984;47:1307-13.

${ }^{2}$ Wiberg J, Nornes H. Effects of carotid endarterectomy en? blood flow in the internal carotid artery. Acta Neurocterr (Wien) 1983;68:217-26.

${ }^{3}$ Wood EH, Correll JW, Bochenstein FK, Reilly A, Sa砶음 JN. Neuroradiologic evaluation of results of surgi@al treatment of extracranial atherosclerotic disease. Ra政- T ology 1969;9:537-48.

${ }^{4}$ Schutz H, Fleming JFR, Awerbuck B. Arteriograp assessment of carotid endarterectomy. Ann Sing 1970;171:509-21.

${ }^{5}$ Blaisdell FW, Lim R jr, Hall AD. Technical results of cartotid endarterectomy. Arteriographic assessment. Am $J$ T Surg 1967;114:239-46.

${ }^{6}$ Rosental JJ, Gaspar MR, Movius HJ. Intraoperative arteriography in carotid thromboendarterectomy. Arch Surg 1973;106:806-8.

${ }^{7}$ Andersen CA, Collins GJ, Rich NM. Routine operative $\frac{0}{\varnothing}$ arteriography during carotid endarterectomy. A reas- $\mathrm{Q}$ sessment. Surgery 1978;83:76-83.

${ }^{8}$ Bodily KC, Zierler RE, Marimelli MR, Thiele BL, Green $Э$ FM jr, Strandness DE jr. Flow disturbances following? carotid endarterectomy. Surg Gynecol Obstet: 1980;151:77-80.

${ }^{9}$ Holder J, Binet EF, Flanigan S, Ferris EJ. Arteriography after carotid endarterectomy. AJNR 1981;2:325-9.

${ }^{10}$ Cantelmo NL, Cutler BS, Wheeler HB, Herrmann JB, Cardullo PA. Noninvasive detection of carotid stenosis following endarterectomy. Arch Surg 1981;116:1005-8.

${ }^{11}$ Hertzer NR, Beven EG, Modic MT, O'Hara PJ, Vogt DP,, Weinstein A. Early patency of the carotid artery after endarteretomy: Digital subtraction angiography after? two hundred and sixty-two operations. Neurosurgery $\frac{D}{O}$ 1982;92:1049-57.

${ }^{12}$ Padayachee TS, Lewis RR, Yates AK, Gosling RG.Ñ Doppler ultrasound assessment of the internal carotid. artery following carotid endarterectomy. Stroke $\mathcal{O}^{-}$ 1983;14:990-4. 